\title{
Anciano Frágil y Calidad de Vida
}

\author{
Ramón Orueta Sáncheza, Angela Rodríguez de Cossíoa, Javier Carmona de la Morenaa,

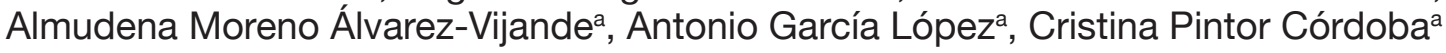

\begin{abstract}
${ }^{a}$ Médico de Familia. Centro de Salud "Sillería" de Toledo.
\end{abstract}

\section{Correspondencia:} Ramón Orueta Sánchez. Centro de Salud "Sillería", C/ Sillería s/n, 45001-Toledo. E-mail: roruetas@papps.org.

Recibido el 29 de noviembre de 2007.

Aceptado para su publicación el 20 de enero de 2008

\section{RESUMEN}

Objetivo. Conocer el grado subjetivo de deterioro de la calidad de vida en ancianos con criterios de fragilidad.

Diseño. Estudio observacional, descriptivo, transversal.

Emplazamiento. Atención Primaria, Zona de Salud.

Participantes. Ancianos con criterios de fragilidad. Criterio de exclusión: ancianos frágiles con deterioro físico/psíquico que impidiera cumplimentar los cuestionarios o negativa a participar (total $8,5 \%)$.

Mediciones principales. Cuestionario de calidad de vida Nottingham Health Profile (NHP) para la variable principal. Otras variables recogidas: sociodemograficas (edad, sexo y convivencia), número de enfermedades crónicas y de fármacos consumidos y el cuestionario de capacidad funcional de Barthel.

Resultados. Edad media de 81,4 años, 67,5\% mujeres, 33,7\% vivían solos, número medio de 3,2 patologías crónicas y de 4,7 fármacos consumidos. El porcentaje de deterioro global en el NHP fue de 25,6 (IC95\% 22,8-28,3), siendo dicho deterioro variable en cada una de las dimensiones: energía $(26,1 ; 21,2-31,0)$, dolor $(20,5 ; 16,8-24,3)$, movilidad $(32,5 ; 28,6-36,5)$, reacción emocional $(23,7$; $20,4-27,1)$, sueño $(33,8 ; 29,0-38,5)$ y aislamiento social $(17,2 ; 14,3-20,1)$. El grado de deterioro global se asoció, de forma estadísticamente significativa, con mayor edad, convivencia fuera del núcleo familiar, mayor número de patologías crónicas, mayor consumo de fármacos y menor capacidad funcional.

Conclusiones. Existe un grado de deterioro subjetivo importante en la calidad de vida del anciano frágil. Parece justificada la inclusión de la valoración de la calidad de vida subjetiva en la valoración integral de este tipo de pacientes.

Palabras clave. Anciano frágil. Calidad de vida.

\section{ABSTRACT}

Frail elderly people and Quality of life

Objective. To determine the subjective deterioration in quality of life in elderly people who met frailty criteria.

Design. Cross-sectional, descriptive, observational study.

Setting. Primary Care, Health Area

Participants. Elderly people with signs of frailty. Exclusion criteria: frail elderly people who, due to their physical or mental impairment, were unable to complete the questionnaires or did not want to take part (total $8.5 \%$ ).

Main measurements. Primary variable: Nottingham Health Profile (NHP) quality of life questionnaire. Other variables were: socio-demographic data (age, gender and co-residence), number of chronic diseases, number of drugs being taken, and Barthel's index for functional capacity.

Results. The mean age of the participants was 81.4 years, $67.5 \%$ were women and $33.7 \%$ lived alone. The mean number of chronic diseases was 3.2 and the mean number of drugs being taken was 4.7 . The percentage of overall deterioration on the NHP was 25.6 (95\% Cl 22.8-28.3), This percentage deterioration varied among the dimensions: energy $(26.1 ; 21.2-31)$, pain $(20.5 ; 16.8-24.3)$, physical mobility $(32.5$; 28.6$36.5)$, emotional reactions $(23.7 ; 20.4-27.1)$, sleep $(33.8 ; 29-38.5)$ and social isolation $(17.2 ; 14.3-20.1)$. The degree of overall deterioration was associated, in a statistically significant manner, with older age, co-residence outside the family, higher number of chronic diseases, higher number of drugs being taken and lower functional capacity.

Conclusions. There is a significant level of subjective deterioration in the quality of life in frail elderly people. Including the subjective evaluation of quality of life in the overall assessment of this type of patient would seem justified.

Key Words. Frail elderly. Quality of life. 


\section{INTRODUCCIÓN}

Desde el pasado siglo nuestro país, al igual que el resto de los países occidentales de nuestro entorno, ha presentado un envejecimiento progresivo de su población debido principalmente a un aumento significativo de la esperanza de vida y a una disminución importante de la fecundidad, lo que hace que las previsiones para España, en caso de no modificarse las tendencias actuales, sitúen por encima del 30\% la población mayor de 65 años para el año $2050^{1-2}$ y que la presencia de personas con edades extremas haya dejado de ser una excepción.

Se define al anciano fragíl como aquél que presenta un aumento de la vulnerabilidad ante la presencia de estresores, como consecuencia de la disminución o la alteración de sus reversas fisiológicas ${ }^{3-4}$, estando implicados criterios socio-demográficos (edad, soporte social, etc.), médicos (pluripatología, hospitalización y/o reingresos, deficit sensoriales, etc.) y funcionales (actividades básicas e instrumentales) ${ }^{3,5-6}$.

Por otra parte, en las ultimas decadas los objetivos sanitarios han pasado de centrarse exclusivamente en la reducción de la morbimortalidad a valorar de forma significativa la calidad de vida de los pacientes ${ }^{7-8}$, estando ésta definida por la Organización Mundial de la Salud (OMS) como la percepción personal de un individuo de su situación en la vida, dentro del contexto cultural y de valores en que vive, y en relación con sus objetivos, expectativas, valores e intereses ${ }^{9}$. Su medición ha permitido pasar de los indicadores negativos de salud clasicos a otros sobre aspectos subjetivos que se centran en el paciente y su entorno desde una vertiente más positivista ${ }^{10}$. Además, pese a tratarse de un componente subjetivo, la medición de la calidad de vida subjetiva es considerada como un instrumento valido para medir el estado de salud de los pacientes, ya que diversos estudios han permitido relacionar dicha calidad de vida con la utilización de servicios sanitarios, el grado de autonomía y/o institucionalización del paciente e incluso con su supervivencia $^{11-13}$.

En este contexto se presenta el siguiente trabajo, cuyos objetivos principales son determinar el grado de deterioro subjetivo en su calidad de vida que presentan nuestros ancianos frágiles y las variables asociadas a dicho deterioro.

\section{MATERIAL Y MÉTODO}

Para lograr los objetivos anteriormente mencionados se realizó un estudio observacional-descriptivo de corte transversal (figura 1), teniendo su ubicación en un centro de caracteristicas urbanas (Centro de Salud "Sillería" de Toledo) y realizandose las encuestas durante los últimos meses de 2006 y primeros meses de 2007.

La población diana fueron todos los ancianos de tres cupos de Medicina de Familia de dicho centro y el criterio de inclusión fue reunir uno o más criterios de anciano frágíl. Se definio como anciano frágíl todo aquel que reuniera al menos uno de los criterios más ampliamente aceptados para su definición: edad igual o superior a 80 años, vivir solo, pérdida reciente de la pareja (inferior a 1 año), padecer patología crónica invalidante (cardiopatia isquémica, demencia, deficit visual y/o auditivo importante, etc.), polifarmacia (consumo de 4 o más fármacos), caídas recientes $y / o$ frecuentes, ingreso hospitalario en el último año y deficiencia económica y/o social3,6,14. Como criterios de exclusión se utilizaron la negativa a participar en el estudio o la presencia de deterioro físico y/o psiquico que impidiera cumplimentar los cuestionarios.

De las historia clínica de cada paciente fueron recogidos datos sociodemograficos (edad, sexo, convivencia, etc.) y clínicos (patologías y consumo de fármacos). A través de una citación especifica en el Centro de Salud o en el domicilio del paciente le fueron administrados el cuestionario de Barthel para capacidad funcional de actividades básicas y el cuestionario de calidad de vida Nottinghan Health Profile (NPH).

El NHP es un cuestionario genérico de calidad de vida relacionada con la salud de carácter autoaplicado o heteroaplicado, influido por el Sickness Impact Profile, que consta de 38 preguntas de respuesta "Si-No" que exploran seis dimensiones de salud: energía, dolor, movilidad física, reacciones emocionales, sueño y aislamiento social ${ }^{15-17}$ y del que existe una versión validada al castellano ${ }^{18-19}$.

Los datos recogidos fueron introducidos en una base de datos del paquete estadistico R-Sigma creada para tal fin y fueron tratados con herramientas de estadistica descriptiva y analitica.

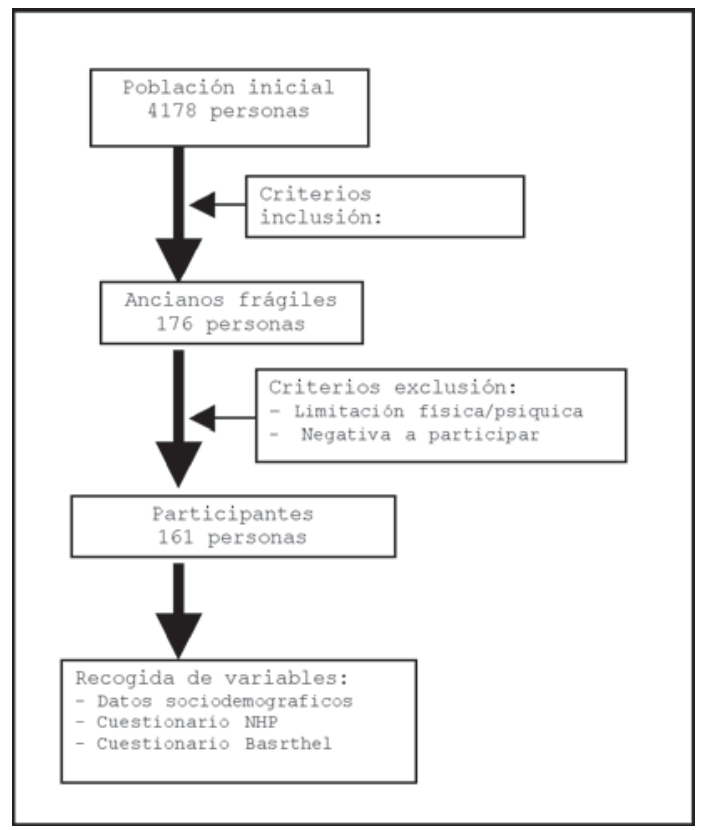

Figura 1. Esquena general del estudio 


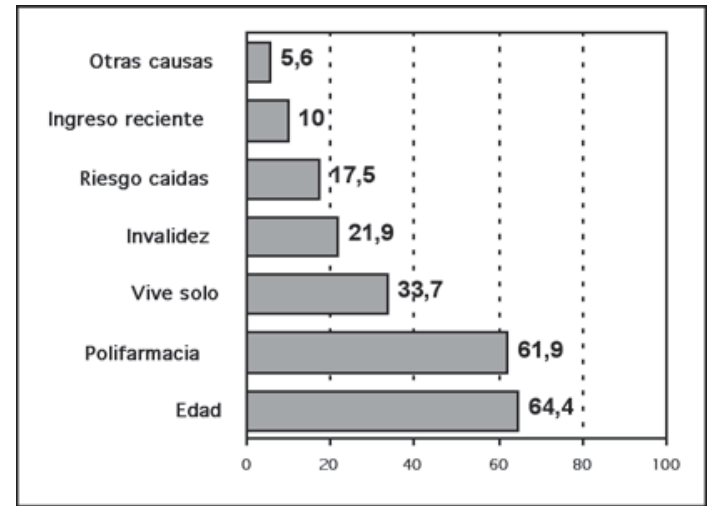

Figura 2. Criterios de Fragilidad.

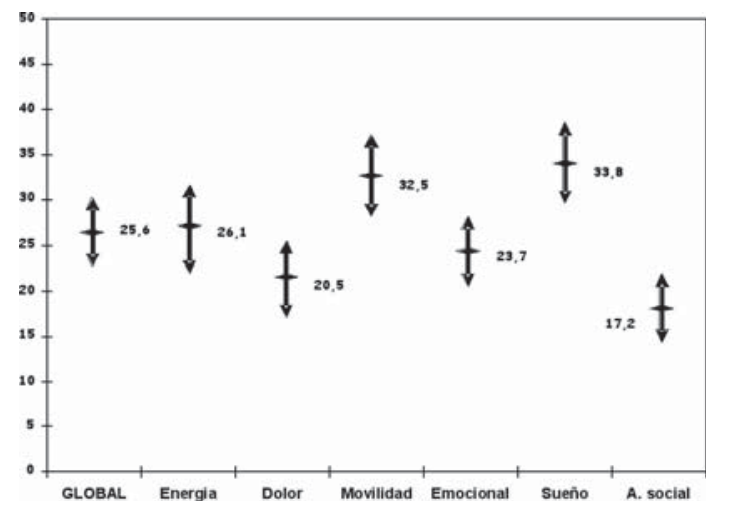

Figura 3. Porcentaje de deterioro.

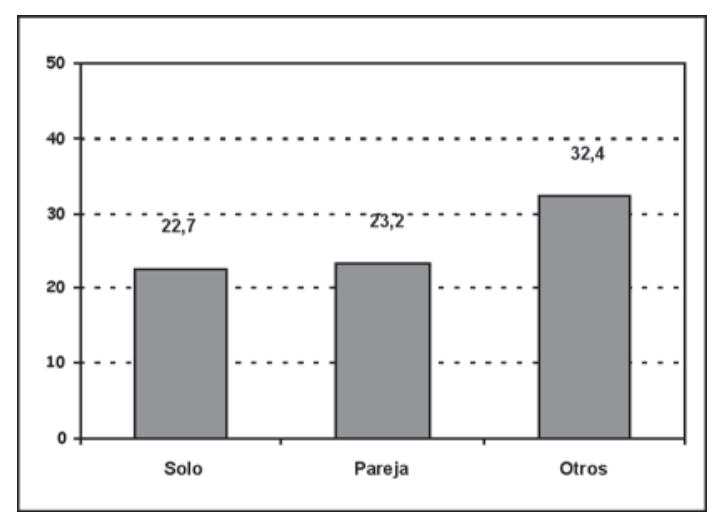

Figura 4. Relación deterioro calidad de vida/convivencia.

\section{RESULTADOS}

En total fueron encontrados 176 pacientes con criterios de inclusión en el estudio, de los que fueron desechados $15(8,5 \%)$ por presentar alguno de los criterios de exclusión mencionados en el apartado anterior, siendo la muestra final de 161 pacientes. Los criterios de fragilidad que reunían los pacientes finalmente incluidos aparecen recogidos en la figura 2.

De los pacientes finalmente incluidos, el $67,5 \%$ eran mujeres, su edad media fue de 81,4 años (DE 4,4 años) y respecto a la convivencia, el $33,7 \%$ vivían solos, el $38,7 \%$ vivián con su pareja y el resto $(27,5 \%)$ con algún otro familiar o colectivo. El número me-

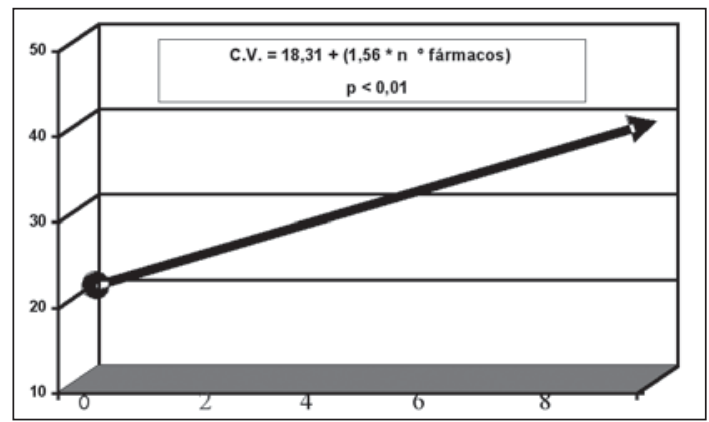

Figura 5. Calidad de vida / $n^{\circ}$ de fármacos.

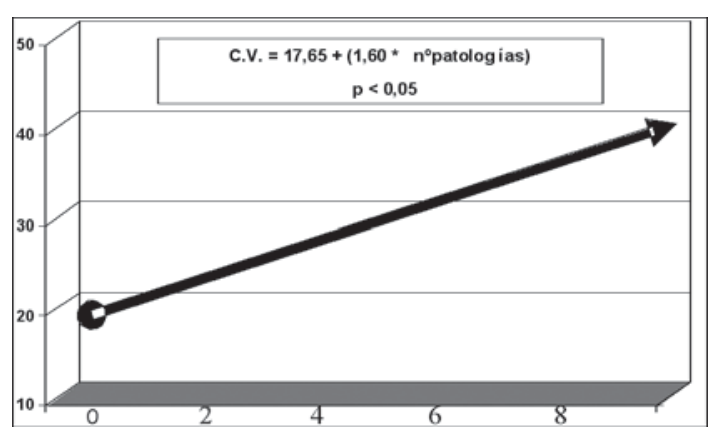

Figura 6. Calidad de vida / $n^{\circ}$ de patologías.

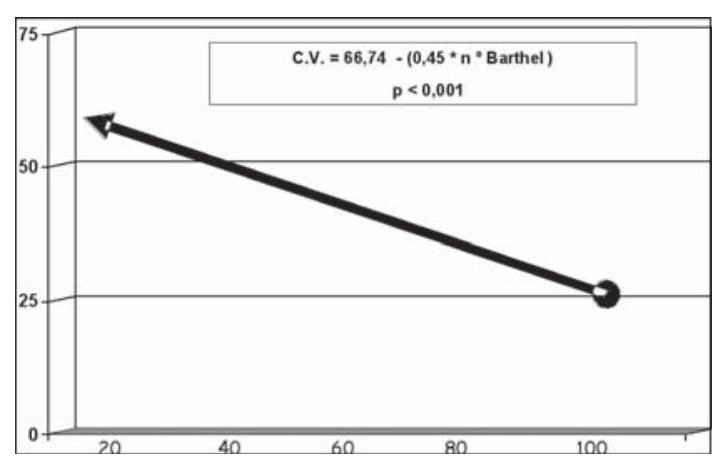

Figura 7. Calidad de vida / Barthel.

dio de patologías crónicas padecidas fue de 3,2 (DE $1,3)$, siendo las más prevalentes hipertensión arterial $(75,5 \%)$, artrosis $(39,4 \%)$, dislipemia $(32,9 \%)$ y diabetes mellitus $(22,6 \%)$. El número medio de fármacos consumidos de forma crónica era de 4,7 (DE 1,4).

El porcentaje de deterioro en la puntuación global del NPH encontrado fue del 25,6 (IC95\% 22,8-28,3). El porcentaje de deterioro, así como sus intervalos de confianza en cada una de las seis dimensiones medidas por el cuestionario, aparece recogido en la figura 3.

El deterioro global de la calidad de vida (DGCV) se asoció de forma directa a la edad (DGCV $=-32,345+$ $\left.\left(0,712^{*} E d a d\right) ; F=5,044 ; p<0,05\right)$, pero no con el sexo del paciente $(23,1$ en hombres y 26,8 en mujeres; $T$ Student $=-1,252 ; p=0,210)$. La figura 4 recoge la relación entre el DGCV y la convivencia del paciente. 
Las figuras 5 y 6 recogen, respectivamente, la relación entre el DGCV y el número de enfermedades crónicas padecidas o el número de fármacos consumidos. La figura 7 recoge la relación del DGCV con el deterioro funcional valorado a través de la escala de Barthel.

\section{DISCUSIÓN}

Prueba del interes de la valoración de la calidad de vida subjetiva es el alto número de publicaciones que el tema genera a nivel internacional ${ }^{20}$ y el haber traspasado el campo sanitario con irrupción en otros sectores como el politico, el social o el economico ${ }^{21}$.

La elección del NHP en nuestro estudio para dicha evaluación vino determinada por tratarse de un instrumento genérico que permite evaluar distintas áreas, con independencia de las patologías padecidas. Por sus caracteristicas, brevedad y comprensibilidad, está especialmente recomendado en población anciana y en estudios de tipo transversal ${ }^{22-24}$, caracteristicas ambas que reune nuestro estudio. Su elección frente a otras herramientas génericas, como los cuestionarios SF-36, EuroQol 5-D o perfil de consecuencias de la enfermedad o las laminas COOP-WONCA ${ }^{25-29}$, también estuvo motivada por tratarse del instrumento de mediada utilizado en un mayor número de estudios comparables al mismo en nuestro entorno ${ }^{30-36}$.

Dado que se trata de un estudio realizado en una población circunscrita, su validez externa no queda totalmente garantizada y debe ser tomada con precaución. Así mismo, la comparabilidad con otros estudios debe ser valorada con prudencia, dado que cada uno de los mismos parte de unos criterios de inclusión y exclusión diferentes.

El porcentaje de detrioro subjetivo global encontardo en nuestro estudio, si bien se tratra de un dato del cuestionario validado como tal, se encuentra en el rango de los obtenidos por otros autores que también lo han valorado ${ }^{31,34-36}$. Asi mismo, los datos encontrados en cada una de las seis dimensiones valoradas son similares a los diferentes estudios, existiendo cierta uniformidad en encontar el mayor deterioro en la esfera de alteraciones del sueño y el menor deterioro en la esfera de apoyo social ${ }^{30-36}$.

No se observaron diferencias significativas respecto al sexo, aunque sí porcentuales, dato que contrasta con otros trabajos donde detectan un mayor deterio-

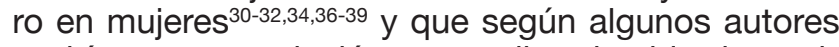
podría estar en relación con estilos de vida determinados, niveles de expectativas diferenciados, mayor aceptación social de la expresión de sus quejas, mayor exposición a problemas emocionales (por ejemplo viudedad, vivir solo, etc.) y menores oportunidades de mejora ${ }^{40-41}$.

El progresivo deterioro en función de la edad es concordante con el aumento de dicho deterioro en función del número de patologías crónicas padecidas, del mayor número de fármacos consumidos y de la disminución de la capacidad funcional. Dichas relaciones tambíen son encontradas en nuestro estudio y en los estudios similares ya mencionados en los parrafos anteriores y que vienen a corroborar la estrecha relación entre calidad de vida subjetiva y datos subjetivos como la presencia de patologias crónicas, la limitación funcional o la presencia de discapaci$\operatorname{dad}^{34,37,42-43}$.

Concluimos con la constatación de que existe un grado de deterioro subjetivo de la calidad de vida de nuestros ancianos frágiles y de que dicho deterioro se relaciona con distintas variables tanto sociodemograficas como sanitarias. Por todo ello, coincidimos con diversos autores que plantean la necesidad de incluir la percepción subjetiva de la calidad de vida en el marco de la valoración integral a realizar a cada uno de nuestros ancianos frágiles ${ }^{30,37,44}$.

\section{BIBLIOGRAFÍA}

1. Cantalapiedra M. El envejecimiento demógrafico en España. Fuentes Españolas de Estadistica. 2002; 68:26-7.

2. Sancho M. Las personas mayores en España. Informe 2002. Madrid: Instituto de Migraciones y Servicios Sociales; 2002.

3. Rockwood K, Hogan DB, MacKnight C. Conceptualisation and measurement of frailty in elderly people. Drugs Aging. 2000; 17:295-302.

4. Fried LP, Ferrucci L, Darer J, Williamson JD, Anderson G. Untangling the concepts of disability, frailty and comorbidity: Implications for improved targeting and care. J Gerontol Med Sci. 2004; 59:255-63.

5. Valoración geriátrica integral (I). Evaluación del paciente geriátrico y concepto de fragilidad. Anales Sis San Navarra. 1999; 22 (supl 1):41-50.

6. Winograd CH, Gerety MB, Chung M, Goldstein MK, Dominguez F, Vallone R. Screening for fralty: criteria and predictors of outcomes. J Am Geriatr Soc. 1991; 39:778-84.

7. Fernández JA, Hernández R. Calidad de vida: algo más que una etiqueta de moda. Med Clin (Barc). 1993; 101:676-8.

8. Higginson IJ, Carr AJ. Using quality of life measures in the clinical setting. BMJ. 2001; 322:1297-300.

9. WHOQOL Group. Strudy protocol for the World Health Organization Project to develop a quality of life assessement instrument. Qual Life Res. 1993; 2:153-9.

10. Muldoon MF, Barger Sd, Flory JD, Manuck SB. What are quality of life measurements measuring? BMJ. 1998; 316:542-5.

11. Kaplan G, Bavell V, Lusky A. Subjetive state of health and survival in elderly adults. J Gerontol. 1988; 43:1145-205.

12. Grand A, Grosclaude P, Bocquet M, Pous J, Albarede JL. Disability, psichosocial factors and mortality among the elderly in a rural french population. J Clin Epidemiol. 1990; 43:773-82.

13. Redondo A, Guallar P, Banegas JR, Rodriguez-Artalejo F. Gender differences in the utilization of health-care services among the older adult population of Spain. BMC Public Health. 2006; 6:155.

14. Álvarez M, Benítez MA, Espinosa JM, Espinosa JM, Gorroñogoitia A, Martín I et al. Programas básicos de salud número 7. Programa del anciano: Valoración geriátrica. Madrid: Doyma; 2000.

15. Hunt SM, McKenna SP, McEwen J, Williams J, Papp E. The Nottingham Health Profile: Subjetive health Status and medical consultations. Soc Sci Med. 1981; 15:221-9.

16. Hunt SM, McEwen J, McKenna SP. Measuring health Status: A new tool for clinicians and epidemiologists. J R Coll Gen Pract. 1985; 35:185-8. 
17. Bergner M, Bobbitt RA, Carter WB, Gilson BS. The Sickness Impact Profile. Development and final revision of a health status measure. Med Care. 1981; 19:787-805.

18. Alonso, J, Antó JM, Moreno C. "Spanish version of the Nottingham Health Profile: translation and preliminary validity". Am J Public Health. 1990; 80:704-8.

19. Prieto L, Alonso J, Viladrich MC, Antó JM. Scaling the Spanish version of the Nottingham Health Profile: Evidence of limited value of item weight. J Clin Epidemiol. 1996; 49:31-8.

20. Sanders C, Egger M, Donovan J, Tallon D, Frankel S. Reporting on quality of life in randomised controlled trials: bibliografic study. BMJ. 1998; 317:1191-4.

21. Grupo WHOQOL. Evaluación de la calidad de vida 1994. ¿Por que calidad de vida? Grupo WHOQOL. Foro Mundial de la Salud. Ginebra: Organización Mundial de la Salud; 1996.

22. McDowell I, Newell C. Measuring Health. A guide to rating scales and questionnaires. New York: Oxford University Press; 1.996.

23. Hopton JL, Porter AM, Howie JG. A measure of perceived health in evaluating general practice: the Nottingham Health Profile. Fam Pract. 1991; 8:253-60.

24. Haywood KL, Garratt AM, Fitzpatrick R. Quality of life in older people: a structured review of generic self-assessed health instruments. Qual Life Res. 2005; 14:1651-68.

25. Badia X, Salamero M, Alonso J. La medida de la salud. Guía de escalas de medición en español. $2^{\mathrm{a}}$ ed. Barcelona: Edimac; 1999.

26. Ware JE, Sherbourne CD. The MOS 36-items Short-From Halth Survey (SF-36). Conceptual framework and item selection. Medical Care. 1992; 30(6):473-83.

27. Euroqol Group. Euroqol, a new facility for the measurement of Health Related Quality of Life. Health Policy. 1990; 199-208.

28. Bergner M, Bobbitt RA, Carter WB, Gilson BS. The Sickness Impact Profile. Development and final revision of a health status measure. Med Care. 1981; 19:787-805.

29. Scolten JHG, Van Weel C. Functional status assessment in family practice. The Darmount COOP Fuctional Health Assessment Charts / WONCA. Lelystad: Meditekst; 1992. p. 17-51.

30. Bayo J, Fernandez MC, Orfila F, Dalfó A, Casajuana J, Vila MA et al. Autopercepción de salud y evaluación integral del paciente anciano en un centro de atención primaria. Aten. Primaria. 1996; 17:273-9.

31. Casado JM, González N, Moraleda S, Orueta R, Carmona J, Gómez-Calcerrada RM. Calidad de vida relaciona con la salud en pacientes ancianos en atención primaria. Aten Primaria. 2001; 28:167-73.
32. Azpiazu M, Cruz A, Villagrasa JR, Abanades JC, García N, Alvear F. Factores asociados a mal estado de salud percibido o a mala calidad de vida en personas mayores de 65 años. Rev Esp Salud Pública. 2002; 76:683-99.

33. Azpiazu M, Cruz A, Villagrasa JR, Abanades JC, García N, Alvarez de Mon C. Calidad de vida en mayores de 65 años no institucionalizados de dos áreas sanitarias de Madrid. Aten Primaria. 2003; 31:285-94.

34. Orfila F, Ferrer M, Lamarca R, Tebe C, Domingo-Salvany A, Alonso J. Gender differences in health-related quality of life among the elderly: the role ob objective functional capacity and chronic conditions. Soc Sci Med. 2006; 63:2367-80.

35. García AM, Leiva F, Martos F, García AJ, Prados D, Sánchez de la Cuesta F. Calidad de vida en pacientes en hipertensión arterial y diabetes mellitus tipo 2. Medicina de Familia (And). 2001; 2:29-34.

36. Fernández AM, Bujalance MJ, Leiva F, Martos F, García AJ, Sánchez de la Cuesta F. Salud autopercibida, apoyo social y familiar de los pacientes con enfermedad pulmonar obstructiva crónica. Medifam. 2001; 11:530-9.

37. Seculi E, Fusté J, Brugulat P, Juncá S, Rué M, Guillén M. Precepción del estado de salud en varones y mujeres en las últimas etapas de la vida. Gac Sanit. 2001; 15:217-23.

38. Rubio R, Aleizxandre M, Cabezas JL. Calidad de vida y diferencias en función del sexo. Rev Mult Gerontol. 2001; 11:173180.

39. Farré R, Frasquet I, Roma I. Estado de salud y calidad de vida de un colectivo de ancianos institucionalizados. Rev Esp Geriatr Gereontol. 1999; 34:25-33.

40. Arber S, Ginn, J. Relación entre género y envejecimiento. Madrid: Narcea; 1996.

41. Lizán L, Reig A. Prespectiva del paciente en la evaluación de resultados en atención primaria: la medida de la calidad de vida relacionada con la salud. Cuadernos de Gestión. 1998; 4:11931.

42. Ferrer M, Lamarca R, Orfila F, Alonso J. Comparison of performance-based and self-rated functional capacity in Spanish elderly. Am J Epidemiol. 1999; 149:228-35.

43. Grimby A, Svamborg A. Morbidity and health-related quality of life among ambulant elderly citizens. Aging (Milano). 1997; 9: 356-64.

44. Díaz A, Nadal MJ, Hernández A, Hita A, Magro R. Calidad de vida autopercibida y satisfacción de los sujetos de 40 a 85 años en el Area de Salud de Guadalajara. Rev Clin Med Fam. 2005; 1:29-33. 